\title{
Non-steroidal anti-inflammatory drugs and gastrointestinal damage-problems and solutions
}

\author{
R I Russell
}

Non-steroidal anti-inflammatory drugs (NSAIDs) have been used for many years for analgesic, anti-inflammatory, and more recently in the case of aspirin, antithrombotic purposes. The use of NSAIDs continues to increase; over 22 million prescriptions are written every year in the UK, and over 70 million in the US. These figures underestimate their full use as aspirin and other NSAIDs are widely available as "over-the-counter" preparations.

The use of these drugs can also be expected to increase in the years to come, partly because of the increasing age of the population and partly because of possible new and developing indications, particularly in vascular disease and cancer prevention. It is therefore of importance to assess the safety and side effects of NSAIDs, and to consider how their safety may be improved. It has long been known that NSAIDs may have a range of side effects, of which the commonest are gastrointestinal.

It is the purpose of this paper to examine the nature, range, and causation of the gastrointestinal side effects associated with the use of NSAIDs, and to consider how these may be reduced or modified. NSAIDs may also be associated with other adverse effects, although these are much rarer than gastrointestinal problems; these include nephropathies, skin rashes, and hepatitis. NSAIDs may also interact with other drugs such as antihyperglycaemic or antihypertensive agents.

\section{Gastrointestinal problems}

NSAIDs may be associated with many gastrointestinal problems, ranging from mild to severe dyspeptic symptoms, the development of gastric or duodenal ulceration, haemorrhage or perforation, and other events which may lead to hospitalisation or death.

Endoscopic studies have shown a prevalence rate of $14 \%-25 \%$ of gastric and duodenal ulcers in NSAID users, ${ }^{1}$ although the difficulty of having accurate control groups makes exact figures difficult to obtain. Although endoscopic studies tend to show more gastric than duodenal ulcers associated with NSAID use, patients presenting with gastrointestinal bleeding on NSAIDs may have a similar frequency of gastric and duodenal ulceration. Dyspeptic symptoms occur in up to $60 \%$ of patients taking NSAIDs and there is a poor correlation between symptoms and endoscopically proved lesions; up to $50 \%$ of endoscopically confirmed ulcers associated with NSAIDs are asymptomatic.

It has been estimated that the individual risk of hospitalisation with gastrointestinal complications of NSAIDs is between $1 / 50$ and $1 / 150$ patient years. ${ }^{2}$ The risk of an upper gastro-

\author{
Box 1: Areas of the gastrointestinal \\ tract that may be damaged by NSAIDs \\ - Oesophagus: oesophagitis, ulceration, \\ stricture \\ - Stomach: ulcers, erosions \\ - Duodenum: ulcers, erosions \\ - Small intestine: ulcers, erosions, protein \\ loss, strictures \\ - Colon: non-specific colitis, exacerbation \\ of ulcerative colitis and Crohn's disease
}

intestinal bleed is between $1 / 100$ and $1 / 500$ patient years; the risk of a gastrointestinal related death is between $1 / 1000$ and $1 / 5000$ patient years.

The risk of serious ulcer complications in patients on NSAIDs has been calculated in various studies, the relative risk (RR) for a serious gastrointestinal event overall being reported as 2.74 in a large meta-analysis, upper gastrointestinal bleeding RR 3.09, perforation RR 5.93, and ulcer related death RR 7.62. ${ }^{3}$

NSAIDs may have adverse effects in all parts of the gastrointestinal tract, not only the stomach or duodenum; the oesophagus, small intestine and colon may also be affected (box 1).

A recent necropsy study of 713 patients has been reported, of whom 244 had NSAIDs prescribed during the six months before death and 464 had not; death in all patients was unrelated to NSAID use. Ulcers of the stomach or duodenum were found in $21.7 \%$ of patients on NSAIDs and $12.3 \%$ of those not on NSAIDs $(\mathrm{p}<0.001)$, and small intestinal ulceration was found in $8.4 \%$ of NSAID users and $0.6 \%$ of patients not on NSAIDs $(p<0.001) .{ }^{4}$ Damage to the small intestine in NSAID users has also been demonstrated at enteroscopy. Patients with small intestinal damage associated with chronic NSAID use may present with chronic iron deficiency anaemia or hypoalbuminaemia due to blood or protein loss, and may develop overt bleeding, perforation, or strictures.

In the oesophagus, chronic NSAID use may be associated, although rarely, with oesophagitis, ulceration, or stricture formation. ${ }^{5}$

In the large bowel, NSAID use may lead to the development of a non-specific colitis (with abdominal discomfort, bloody diarrhoea, and weight loss). NSAIDs may also cause an exacerbation of ulcerative colitis or Crohn's disease, if the associated arthropathy in inflammatory bowel disease is treated with NSAIDs. ${ }^{67}$

High risk factors for NSAID related gastrointestinal damage include older age group (especially over 70), previous history of peptic ulceration, and probably the first three months of NSAID treatment. Other high risk factors are smoking, underlying respiratory or cardio- 


\section{Box 2: Risk factors for gastrointestinal damage due to NSAIDs \\ - Age: older age group, especially over 70 \\ - Previous ulcer history \\ - First three months of treatment \\ - Smoking \\ - Underlying cardiovascular or respira- tory disease \\ - Concomitant drug use, especially corticosteroids and anticoagulants \\ - Use of NSAIDs in high doses, and more than one NSAID \\ - ?Helicobacter pylori}

vascular disease, and concomitant drug use, particularly corticosteroids, aspirin, and anticoagulants (box 2). ${ }^{8}$ Perioperative use is also a risk factor. Individual NSAIDs incurring highest risk include azapropazone, ketoprofen and piroxicam, and those with least risk include ibuprofen, diclofenac, and etodolac. Higher doses are associated with increased risk and also the use of more than one NSAID. There is also an increased risk of gastrointestinal complications with relatively low dose prophylactic aspirin, which is widely used nowadays. ${ }^{9}$ There is debate on the possible interaction and increased risk of Helicobacter pylori causing ulceration with NSAIDs, and this will be discussed in a later section. The balance of physiological association and clinical evidence tends to support the possibility of some interaction occurring, especially in those patients at high risk and possibly in those who have bled. Well planned, prospective controlled studies are required to provide more information on this with respect to different types and doses of NSAIDs, different forms and strains of $H$ pylori, and different types of erosions and ulcers.

\section{Why do NSAIDs cause gastrointestinal damage?}

NSAIDs interfere with the cyclo-oxygenase (COX) pathways which lead to the production of prostanoids (prostaglandins, prostacycline, and thromboxane). This interferes with mucosal protection by reducing the effectiveness of the mucus-bicarbonate barrier; gastric acid, and possibly also pepsin, are thus more likely to cause damage. The fact that most NSAIDs are also weak acids may also be a contributory factor.

It is now known that COX exists as two separate isoforms, COX-1 and COX-2, which differ markedly in their tissue expression and regulation. $.^{10-12} \mathrm{COX}-1$ is constitutively expressed in most tissues, including the stomach, duodenum, platelets, and kidneys; COX-1 plays a key part in the production of prostaglandins which regulate important physiological processes such as gastrointestinal cytoprotection (maintaining an effective mucusbicarbonate barrier, submucosal blood flow, quicker and more effective mucosal adaptation to initial tissue damage, and more rapid recovery when such damage occurs). It is also involved in vascular homoeostasis and the

\section{Box 3: COX-1 and COX-2}

COX-1

- Constitutively expressed in most tissues: stomach, duodenum, kidneys, platelets

- Key role in the production of prostaglandins which regulate physiological processes: gastrointestinal protection, blood flow, adaptation, cellular recovery, maintenance of good renal function, vascular homoeostasis important "housekeeping" role

COX -2

- Normally undetectable in most tissues

- Can be induced rapidly and in large quantities in the presence of inflammation and other pathological processes

maintenance of good renal function and in maintaining normal physiological functions in many other cells. This is an important "housekeeping" role. COX-2, on the other hand, is normally undetectable in most tissues, but it can be induced rapidly, and in large quantities - to 200-300-fold-by cytokines, growth factors, and hormones in the presence of inflammation and other pathological processes (box 3). Platelets appear to contain only COX-1.

Most conventional NSAIDs are nonselective in their COX inhibition, exerting their anti-inflammatory effects through the inhibition of COX-2, but having adverse effects (such as gastrointestinal mucosal damage and nephrotoxicity) primarily due to inhibition of COX-1. Some existing and longstanding NSAIDs, such as etodolac, a known safer NSAID with respect to gastrointestinal damage and which is known to have reduced adverse effects on mucosal prostaglandins, has been subsequently found to have a degree of COX-2 inhibition selectivity.

Currently, a range of new and specific COX-2 selective inhibitor NSAIDs have been, and are being further developed with the hope of reducing possible gastrointestinal side effects; the preliminary clinical results, as we shall see later, are encouraging. One of these, rofecoxib, is now available in this country.

\section{Solutions}

Treatment and prevention of NSAID related gastrointestinal problems are shown in box 4 .

\section{HEALING OF NSAID RELATED ULCERS}

There is evidence from both animal and human studies that NSAIDs retard the healing of gastric ulcers. Although it is frequently stated that a first step if gastrointestinal problems occur with NSAIDs is to withdraw NSAIDs or reduce the dose, in practical terms this is generally unrealistic, especially when there is a major inflammatory condition such as rheumatoid arthritis.

It may be possible to reduce the dose of NSAIDs in some individuals. It is also appropriate to try alternative conventional NSAIDs as there is a wide variability in responses to individual drugs. Some NSAIDs 


\section{Box 4: Treatment and prevention of NSAID related gastrointestinal problems \\ Healing of NSAID related ulcers \\ - Reduce NSAID dose if possible, or use alternative NSAIDs \\ - Use of acid suppressive drugs while continuing NSAIDs: high dose $\mathrm{H}_{2}$-receptor antagonists, or more effec- tively, proton pump inhibitors \\ Prevention of NSAID related gastrointestinal problems \\ - Avoidance or reduction of risk factors if possible \\ - Drugs which may improve mucosal pro- tection: prostaglandin analogues (misoprostol) \\ - Acid suppressive drugs: $\mathrm{H}_{2}$-receptor antagonists (high doses), proton pump inhibitors \\ - COX-2 specific inhibitors \\ - Eradication of Helicobacter pylori (in selected cases)}

are more likely than others to cause gastrointestinal problems; those associated with greatest risk include azapropazone, ketoprofen, and piroxicam.

$\mathrm{H}_{2}$-receptor antagonists, especially in high doses, do heal NSAID related ulcers while NSAIDs are continued, ${ }^{13}$ but proton pump inhibitors may be expected to be more effective, and have now been shown to be so. Omeprazole has been shown to heal gastric ulcers faster than ranitidine. ${ }^{14}$ In two large studies comparing omeprazole $(20 \mathrm{mg}$ or 40 $\mathrm{mg}$ daily) with misoprostol (200 $\mu \mathrm{g}$ four times a day), the proton pump inhibitors healed significantly more gastric and duodenal ulcers than ranitidine or misoprostol.

No extra benefit was gained from using omeprazole at the higher dose. ${ }^{15} 16$

PREVENTION OF NSAID RELATED

GASTROINTESTINAL PROBLEMS

The lowest dose of the safest NSAID which is effective in individual patients should be used if possible.

Avoidance or reduction of risk factors may be possible in some patients, such as smoking, use in patients with a past history of dyspeptic problems or peptic ulcer, perioperative use, or cotherapy with other drugs such as corticosteroids, anticoagulants, or aspirin. Advice should be given about "over-the-counter" preparations.

There will inevitably be patients in whom additional requirements will be necessary, such as those who continue to require high dose NSAIDs or in those with risk factors which cannot be modified.

In such patients there has been much interest in recent years in attempting to reduce risk by the coprescription with NSAIDs of drugs which may improve mucosal protection (the prostaglandin analogue misoprostol) or reduce gastric acid $\left(\mathrm{H}_{2}\right.$-receptor antagonists or proton pump inhibitors). More recently, newer
NSAIDs-selective COX-2 inhibitors-offer the prospect of greater gastrointestinal safety.

PROSTAGLANDINS

The prostaglandin analogue misoprostol has shown a reduction in ulceration compared with placebo ranging from $50 \%$ to $90 \%$ over $3-12$ months; the dose used initially was $200 \mu \mathrm{g}$ four times a day and subsequently 400-600 $\mu \mathrm{g}$ per day. Protection has been generally similar for both gastric and duodenal ulcers. ${ }^{17}{ }^{18} \mathrm{~A}$ large blinded study of misoprostol compared with placebo (the "MUCOSA" study) reported a significant reduction of gastrointestinal bleeding and perforation with misoprostol at $800 \mu \mathrm{g}$ daily. ${ }^{19}$ In this study, $27 \%$ of patients withdrew because of side effects, principally diarrhoea. Diarrhoea remains a problem for many patients with this preparation. Misoprostol has also been combined with a standard NSAID, diclofenac (arthrotec), and this has proved popular with a number of patients.

Theoretically, prostaglandin analogues have an advantage over acid suppressants in that they should provide mucosal protection throughout the gastrointestinal tract.

ACID SUPPRESSION

As gastric acid is a factor in causing gastroduodenal damage associated with NSAID use, reduction of gastric acid by the coprescription of acid suppressant drugs has been studied.

The use of $\mathrm{H}_{2}$-receptor antagonists in standard doses for the prevention of NSAID associated ulcers has shown some protection against duodenal ulcers. ${ }^{20}$ and the use of high dose famotidine ( $40 \mathrm{mg}$ twice a day) has been shown to significantly reduce the cumulative incidence of both gastric and duodenal ulcers in patients with rheumatoid arthritis on long term NSAID therapy. ${ }^{21}$

Proton pump inhibitors cause more effective acid suppression than $\mathrm{H}_{2}$-receptor antagonists, and several randomised controlled trials lasting 3-6 months using omeprazole $20 \mathrm{mg}$ daily have shown efficacy in preventing both gastric and duodenal ulcers while continuing long term NSAIDs, with about a $75 \%-80 \%$ reduction in ulcers compared with placebo. ${ }^{15} 1622$

The proton pump inhibitors were shown in two of these studies to provide more effective protection than ranitidine ( $150 \mathrm{mg}$ twice a day) or misoprostol (200 $\mu \mathrm{g}$ twice a day).

Omeprazole $20 \mathrm{mg}$ daily has also been shown to protect against bleeding from ulcers in long term NSAID users. ${ }^{23}$

NEWER NSAIDS: COX-2 SPECIFIC INHIBITORS

The search for safer NSAIDs has recently focused on the development of preferential or selective COX-2 inhibitors. These compounds aim to exploit the belief that COX-1 is associated predominantly with the production of protective prostaglandins and has a "housekeeping" role, whereas COX-2 is induced in inflammation and associated with inflammatory processes. The development of these concepts has been recently reviewed. ${ }^{24}$

The definition of, and screening for, COX-2 selectivity has given rise to much debate, 
involving a variety of suggested systems that includes purified recombinant enzyme, transfected cells, and whole blood assays. The latter method may be the most appropriate at present, but all drugs suggested as COX-2 specific inhibitors require to be shown to have no significant inhibition of gastric mucosal prostaglandins, in addition to a good clinical safety profile and effectiveness.

Conventional longstanding NSAIDs have been found to vary with respect to their relative COX-1 to COX-2 inhibition capacity, but all have a significant effect on COX-1, thus interfering with gastric mucosal protection.

One NSAID, etodolac, which has been used widely for some years, has been shown in several studies to be clinically effective and have reduced gastrointestinal toxicity, demonstrated endoscopically and clinically; these studies also found no significant reduction of gastric mucosal prostaglandins compared with naproxen. ${ }^{25}{ }^{26}$ Etodolac has since been found to have some degree of COX-2 selectivity. ${ }^{27}$

Other drugs with probable relative COX-2 selectivity include nabumetone, nimesulide, and meloxicam. ${ }^{24}$

Newer more specific COX-2 inhibitors currently under study include celecoxib and rofecoxib (the latter now being available in this country). Preliminary results of studies with these drugs indicate effectiveness and a good gastrointestinal safety profile. Celecoxib (25$400 \mathrm{mg}$ twice a day) was associated with a significantly reduced incidence of adverse upper gastrointestinal problems including ulcers, gastrointestinal bleeding, and perforation in patients with osteoarthritis and rheumatoid arthritis compared with standard NSAIDs. ${ }^{28}$ Rofecoxib (12.5-25 mg a day) demonstrated a lower incidence of endoscopic ulcers compared with ibuprofen in patients with osteoarthritis in a multicentre study over 24 weeks. ${ }^{29}$ Rofecoxib has also shown reduced occurrence of ulcers, perforations, and gastrointestinal bleeding.

COX-2 specific inhibitors have little, if any, effect on platelet function, and may therefore be associated with a marked reduction of gastrointestinal bleeding associated with NSAID use.

These studies are promising and more data are awaited. The long term safety of these drugs remains to be established, particularly with respect to the kidney and in the possible, although less likely, impairment of healing of pre-existing peptic ulcers. It is also theoretically possible that COX-2 inhibitors may have a benefit in preventing colon cancer and possibly also in Alzheimer's disease. COX-2 inhibitors will not be effective in cardiovascular protection unlike aspirin, as the protective effects provided by aspirin are mediated through COX-1

\section{LOW DOSE ASPIRIN}

Aspirin is widely used in low doses in patients with suspected or definite vascular disease. Aspirin is a major cause of upper gastrointestinal problems, and is a frequent cause of upper gastrointestinal bleeding. Even in low doses (75 mg daily), aspirin has effects on platelet levels of COX, which it acetylates and to which it binds irreversibly, thus impairing platelet function. This causes changes in the bleeding time, platelet aggregation, and the synthesis of thromboxane, which remain for the life of the platelet. The damage is worse with longer acting NSAIDs with long half lives and an extensive enterohepatic circulation, such as piroxicam.

With increasing use, aspirin is now a major cause of upper gastrointestinal problems, and in particular, upper gastrointestinal bleeding. One recent study reported an overall odds ratio of 3.2 (95\% confidence interval (CI) 2.3 to 4.4 ) in gastrointestinal bleeding for daily aspirin use of at least one month, compared with $3.8(95 \%$ CI 3.1 to 4.5$)$ for non-aspirin NSAID use. ${ }^{9}$ This can be expected to increase with increasing use of low dose aspirin, especially common in older age groups.

Platelets contain only COX-1; thus only drugs such as aspirin and those NSAIDs which inhibit COX-1 will inhibit platelet function. COX-1 sparing NSAIDs such as etodolac, nabumetone, nimesulide, meloxicam, are likely to have little effect on platelet dysfunction. The highly specific COX-2 inhibitors (celecoxib, rofecoxib), have almost no effect on platelet function, and are likely, as mentioned previously, to lead to marked reductions in upper gastrointestinal bleeding. However, if used with low dose aspirin, there is likely to be a loss of benefit.

COX-2 specific inhibitors will not be helpful in vascular disease, and cannot be considered as a replacement in this respect for low dose aspirin.

A new antiplatelet agent, clopidrogel may be safer than aspirin, although more costly. Clopidrogel requires to be further assessed, but may be helpful in patients with peripheral artery disease, and in stroke or myocardial infarction in whom aspirin is contraindicated, or in whom aspirin fails to achieve the required therapeutic effect.

\section{WHAT TO DO ABOUT H PYLORI?}

$H$ pylori and NSAIDs are the two most common causes of peptic ulceration.

The possible interactions of $H$ pylori and NSAIDs have led to much discussion recently and no clear picture emerges that can apply to all situations. In many ways, NSAIDs and $H$ pylori have similar adverse effects on mucosal protective mechanisms, and despite $H$ pylori itself producing small amounts of prostaglandins, there remains the possibility of an additive damaging effect when both are present. ${ }^{30}$ Studies on mucosal adaptation and on neutrophils raise the possibility of some inter-relationship that may allow damage to occur more readily when NSAIDs are taken in the presence of $H$ pylori. $^{31}$

The development of an ulcer when NSAIDs are given to patients who are $H$ pylori positive may depend on the interaction of a number of factors, including previous exposure to NSAIDs, past history, gastric acid output, and the use of acid suppression drugs such as proton pump inhibitors. 
Aspirin may have different interactions with $H$ pylori, particularly with respect to the risk of gastrointestinal haemorrhage.

Gastrointestinal haemorrhage from ulcers may be in a different category from nonbleeding ulcers, and really requires to be studied separately; other factors may be involved, such as the possible antiplatelet effects of many NSAIDs, which may be a further factor in bleeding.

Clinical studies in this area have given conflicting results, although one study in patients not previously given NSAIDs clearly demonstrated an advantage in eradicating $H$ pylori before starting NSAIDs; significantly fewer lesions developed over two months in patients in whom $H$ pylori had been eradicated before starting NSAIDs. ${ }^{32}$ In such patients, especially if risk factors are present (older age group, previous ulcer history), eradication of $H$ pylori before starting NSAIDs may be indicated.

A large multicentre study has found that eradication of $H$ pylori did not reduce the rate of ulcer relapse in existing long term NSAID users. ${ }^{33}$ In this study, it was also reported in a subgroup of 41 patients with gastric ulcers found at baseline endoscopy that eradication of $H$ pylori was associated with delayed ulcer healing. However, in another prospective, randomised study of 195 patients with $H$ pylori infection and NSAID associated bleeding ulcers, eradication of $H$ pylori did not impair healing of gastric or duodenal ulcers. ${ }^{34} \mathrm{~A}$ prospective, randomised clinical outcome study compared $H$ pylori eradication alone with long term omeprazole for the prevention of recurrent ulcer haemorrhage in high risk users of aspirin or non-aspirin NSAIDs. ${ }^{35}$ The results to date indicate that eradication of $H$ pylori alone did not prevent recurrent ulcer bleeding associated with non-aspirin NSAIDs, but $H$ pylori eradication alone was as effective as maintenance omeprazole in preventing recurrent haemorrhage associated with low dose aspirin. Perhaps the antiplatelet effects of NSAIDs are relevant there.

$H$ pylori testing would probably not be indicated in patients who have already been on NSAIDs or aspirin for some time without any adverse effects. In patients with a past history of peptic ulcer or dyspepsia on NSAIDs, long term acid suppressive agents such as proton pump inhibitors are indicated even after eradication of $H$ pylori.

More scientific and clinical data are required on these complex inter-relationships.

\section{Conclusions}

- The use of NSAIDs continues to increase, especially in the elderly.

- NSAIDs may have adverse effects in any part of the gastrointestinal tract: oesophagus, stomach, duodenum, small intestine, or colon.

- Risk factors for gastrointestinal damage due to NSAIDs include age, previous ulcer history, first three months of treatment, smoking, underlying cardiovascular or respiratory disease, concomitant drug use with
Questions (answers on p 88)

1. Do patients with NSAID related ulcers always have dyspeptic symptoms?

2. In a patient on long term NSAIDs who has developed chronic iron deficiency anaemia, is the cause always likely to be found in the stomach or duodenum?

3. Is the use of low dose prophylactic aspirin safe with respect to the gastrointestinal tract?

4. Why don't we just reduce the dose of NSAIDs, or even stop NSAIDs, in patients at high risk or with gastrointestinal problems?

5. Is the cost of coprescription of acid suppressive drugs justified in patients on long term NSAIDs?

6. Is it likely that selective COX-2 inhibitors will reduce serious problems such as gastrointestinal haemorrhage?

7. Are patients on corticosteroids in addition to NSAIDs at greater risk of gastrointestinal problems?

8. Have prostaglandin analogues any advantage over acid suppressive agents in preventing NSAID problems in the gastrointestinal tract?

9. In considering risk factors for NSAID related gastrointestinal damage, is there any particular time of greatest danger?

10. Why should specific COX-2 inhibitors be safer?

corticosteroids and anticoagulants, high dose and multiple NSAIDs, and possibly in some cases $H$ pylori.

- Low dose prophylactic aspirin may also be associated with adverse gastrointestinal effects.

- Healing of NSAID related ulcers can be achieved while NSAIDs are continued by the use of $\mathrm{H}_{2}$-receptor antagonists in high doses, or more effectively by proton pump inhibitors.

- Prevention of NSAID related gastrointestinal problems may be achieved by identifying and if possible reducing risk factors, the coprescription of prostaglandin analogues or acid suppressive drugs (especially proton pump inhibitors), or by using the currently being developed and promising COX-2 specific inhibitors.

- The development of COX-2 specific inhibitors offers the hope of real progress in producing much safer and effective NSAIDs.

1 Hudson N, Hawkey CJ. Non-steroidal anti-inflammatory drug associated upper gastrointestinal ulceration an complications. Eur f Gastroenterol Hepatol 1993;5:412-19.

2 McCarthy, DM. Nonsteroidal anti-inflammatory drugs: reducing the risks to the gastrointestinal tract. Clinical Perspectives in Gastroenterology 1999;4:219-26.

3 Gabriel SE, Jaakkimaimen L, Bombardier C. Risk for serious gastrointestinal complications related to the use of non-steroidal anti-inflammatory drugs - a meta-analysis. non-steroidal anti-inflammatory

4 Allison MC, Howatson AG, Torrance CJ, et al. GastroAllison MC, Howatson AG, Torrance CJ, et al. Gastrointestinal damage associated with the use of non-steroid anti-inflammatory drugs. N Engl f Med 1992;327:749-54 Mason JC. NSAIDs and the oesophagus. Eur $\mathcal{F}$ Gastroenterol Hepatol 1999;11:369-73. 
6 Faucheron J-L. Toxicity of non-steroidal anti-inflammatory drugs in the large bowel. Eur $f$ Gastroenterol Hepatol

7 Forrest E, Russell RI. Peripheral arthropathies in inflammatory bowel disease. Gut 1999;44:439.

8 Russell RI. Defining patients at risk of NSAID gastropathy. Ital $f$ Gastroenterol Hepatol 1999;31(suppl 1 S):14-18.
9 Weil J, Colin-Jones D, Langman M, et al. Prophylactic 9 Weil J, Colin-Jones D, Langman M, et al. Prophylactic aspi-
rin and risk of peptic ulcer bleeding. BMf 1995;310:82730.

$10 \mathrm{Fu}$ JY, Masferrer JL, Seibert K, et al. The induction and suppression of prostaglandin $\mathrm{H} 2$ synthase (cyclooxygenase) in human monocytes. F Biol Chem 1990;265: 16737-40.

11 Kujubu DA, Fletcher BS, Varnum BC, et al. TIS 10, a phorbol ester tumor promoter-inducible mRNA from Swiss 3T 3 cells, encodes novel prostaglandin synthase/cyclo-oxygenase homologue. F Biol Chem 1991;266:12866-72.

12 DeWitt DL, Meade EA, Smith WL. Prostaglandin H synthase isoenzyme activity: the potential for safer nonsynthase isoenzyme activity: the potential for safer non-
steroidal anti-inflammatory drugs. Am 7 Med 1993;95:40S6 .

13 Hudson N, Taha AS, Russell RI, et al. Famotidine for healing and maintenance in non-steroidal anti-inflammatory drug associated gastroduodenal ulceration. Gastroenterology 1997;112:1817-2

14 Walan A, Bader JP, Glassen M, et al. Effect of omeprazole and ranitidine on ulcer healing and relapse rates in patients

with benign gastric ulcer. N Engl f Med 1989;320:69-75.

15 Hawkey CJ, Karrasch JA, Szczepanski L, et al. Omeprazole compared with misoprostol for ulcers associated with non-steroidal anti-inflammatory drugs. N Engl f Med 1998 ;
338:727-34.

16 Yeomans ND, Tulassay Z, Juhasz L, et al. Omeprazole compared with ranitidine for ulcers associated with non-
steroidal anti-inflammatory drugs. $N$ Engl F Med 1998;338: 719-26.

17 Graham DY, Agrawal NM, Roth SH. Prevention of NSAID-induced gastric ulcer with misoprostol: multiNSAID-induced gastric ulcer with misoprostol: multi-
centre, double-blind, placebo-controlled trial. Lancet 1988; ii: $1277-80$

18 Graham DY, White RH, Moreland LW, et al and the Misoprostol Study Group. Duodenal and gastric ulcer prevention with misoprostol in arthritis patients taking NSAIDs. Ann Intern Med 1993;119:257-62.

19 Silverstein FE, Graham DY, Senior JR, et al. Misoprostol reduces serious gastrointestinal complications in patient with rheumatoid arthritis receiving non-steroidal antiinflammatory drugs. A randomised, double-blind, placebocontrolled trial. Ann Intern Med 1995;123:241-9.

20 Ehsanullah RSB, Page MC, Tildesley G, et al. Prevention of gastroduodenal damage induced by non-steroidal antiinflammatory drugs: controlled trial of ranitidine. $B M \mathcal{F}$ 1988;297:1017-21.

21 Taha AS, Hudson N, Hawkey CJ, et al. Famotidine for the prevention of gastric and duodenal ulcers caused by non-steroidal anti-inflammatory drugs. $N$ Engl f Med 1996; 334:1435-9.

22 Ekstrom P, Carling L, Wetterhus S, et al. Prevention of peptic ulcer and dyspeptic symptoms with omeprazole patients receiving continuous non-steroidal anti-inflammatory drug therapy. A Nordic multi-centre study. Scand $\mathcal{F}$ Gastroenterol 1996;31:753-8.

23 Chan FKL, Sung JY, Suen R, et al. Eradication of $\mathrm{H}$ pylori versus maintenance acid suppression to prevent recurrent ulcer haemorrhage in high risk NSAID users: a prospective randomised study. Gastroenterology 1998;114:A87.

24 Hawkey CJ. COX-2 inhibitors. Lancet 1999;353:307-14.

25 Russell RI. Endoscopic evaluation of etodolac and naproxen, and their effects on gastric and duodenal prostagnaproxen, and their effects on gastric and duoden

26 Laine L, Sloane R, Ferretti M, et al. A randomised, doubleblind comparison of placebo, etodolac, and naproxen on gastrointestinal injury and prostaglandin production. Gastrointest Endosc 1995;42:428-33.

27 Glaser K. Cyclo-oxygenase activity and NSAIDs: cyclooxygenase-2 selectivity of etodolac (Lodine). Inflammo Pharmacology 1995;3:335-45.

28 Goldstein AL, Agrawal NM, Silverstein F, et al. Celecoxib is associated with a significantly lower incidence of clinically significant upper gastrointestinal events in osteoarthritis and rheumatoid arthritis patients as compared to NSAIDs. Gastroenterology 1999;116A:174.

29 Laine L, Hawkey CW, Harper S, et al. Effect of a COX-2 specific inhibitor Rofecoxib on ulcer formation: a doublespecific inhibitor Rofecoxib on ulcer formation: a double-
blind comparison with ibuprofen and placebo. Gastroenterolblind comparison wi

30 Taha AS, Sturrock RD, Russell RI. Mucosal erosions in long-term non-steroidal anti-inflammatory drug users: predisposition to ulceration and relation to Helicobacter pylori. Gut 1995;36:334-6.

31 Taha AS, Dahill S, Morran C, et al. Neutrophils, Helicobacter pylori, and non-steroidal anti-inflammatory drug ulcers. Gastroenterology 1999;116:254-8.

32 Chan FKL, Sung JJY, Chung C, et al. Randomised trial of eradication of Helicobacter pylori before starting nonsteroidal anti-inflammatory drug therapy to prevent peptic ulcers. Lancet 1997;350:975-9.

33 Hawkey CJ, Tulassay Z, Szczepanski L, et al. Randomised control trial of Helicobacter pylori eradication in patients on non-steroidal anti-inflammatory drugs: HELP NSAIDs study. Lancet 1998;352:1016-21.

34 Chan FKL, Sung JJY, Suen R, et al. Does eradication of $\mathrm{H}$ pylori impair healing of non-steroidal anti-inflammatory drug associated bleeding peptic ulcers? A prospective randomised study. Aliment Pharmacol Ther 1998;12:1201-5.

35 Chan FKL, Sung JJY, Suen R, et al. Prospective randomised trial of $\mathrm{H}$ pylori eradication versus maintenance omeprazole to prevent recurrent upper gastrointestinal haemorrhage in high-risk aspirin and non-aspirin NSAID users. Gastroenterology 2000;118:A194. 


\section{Answers}

1. No: Up to $50 \%$ of endoscopically confirmed ulcers associated with NSAIDs are asymptomatic. Dyspeptic symptoms occur in up to $60 \%$ of patients taking NSAIDs and there is a poor correlation between symptoms and endoscopically proved lesions.

2. No: NSAIDs may have adverse effects in all parts of the gastrointestinal tract, including oesophagus, stomach, duodenum, small intestine, and colon.

A patient with chronic iron deficiency anaemia on long term NSAIDs may well have small intestinal damage associated with NSAIDs, leading to blood loss and chronic iron deficiency anaemia. Overt bleeding, perforation, or strictures of the small intestine may also occur.

3. No: There is an increased risk of gastrointestinal complications with relatively low dose prophylactic aspirin, and overt gastrointestinal haemorrhage may occur. With increasing use of low dose aspirin, there is likely to be increasing problems with gastrointestinal side effects. A high level of awareness of the dangers of low dose prophylactic aspirin is necessary.

4. It is rarely feasible to do so, especially in patients who have been on NSAIDs for some time. Most of these patients have rheumatoid arthritis and require the continuation of NSAIDs for adequate treatment of the disease.

5. Yes: In patients at high risk or who have had gastrointestinal problems with NSAIDs, the use of a prostaglandin analogue or the more expensive acid suppressive drugs such as proton pump inhibitors, is justified in preventing gastrointestinal symptoms and the development of ulceration and possibly gastrointestinal haemorrhage or perforation. The use of COX-2 selective inhibitors may be an important development.

6. Yes: It is likely that this will be the case, although more data are awaited. There is early data suggesting that selective COX-2 inhibitors may reduce the incidence of ulcers, gastrointestinal bleeding, and perforation in patients with rheumatoid arthritis and osteoarthritis. COX-2 specific inhibitors have little effect on platelet function and are likely therefore to be associated with a marked reduction of bleeding.

7. Yes: Such patients have a greatly increased relative risk of adverse effects with NSAIDs. Many patients with long standing rheumatoid arthritis are on corticosteroid therapy in addition to NSAIDs.

8. They may have in that they theoretically provide mucosal protection throughout the gastrointestinal tract. This may be of particular importance in the small intestine. However, many patients treated with the prostaglandin analogue misoprostol develop diarrhoea, and some patients may require to discontinue treatment because of this.

9. Yes: It is considered that the first three months of NSAID therapy is the time of greater risk. This may be related to the ability of the gastrointestinal tract to adapt to NSAIDs after this period.

10. COX exists as two separate isoforms, COX-1 and COX-2. COX-1 is present in most tissues including the gastrointestinal tract and is involved in regulating physiological processes such gastrointestinal mucosal protection, submucosal blood flow, and mucosal adaptation. COX-2 is induced rapidly in most tissues in the presence of inflammation and other pathological processes. Most conventional NSAIDs affect both COX-1 and COX-2, thus interfering with the mucosal protection processes in the gastrointestinal tract. Selective COX-2 inhibitors do not interfere with the protective physiological processes associated with COX-1 and offer the prospect of greater gastrointestinal safety.

It should be noted that COX-2 inhibitors will not be effective in cardiovascular protection as the protective effects provided by aspirin are mediated through COX-1. COX-2 selective inhibitors cannot therefore be used to replace aspirin in this way. 